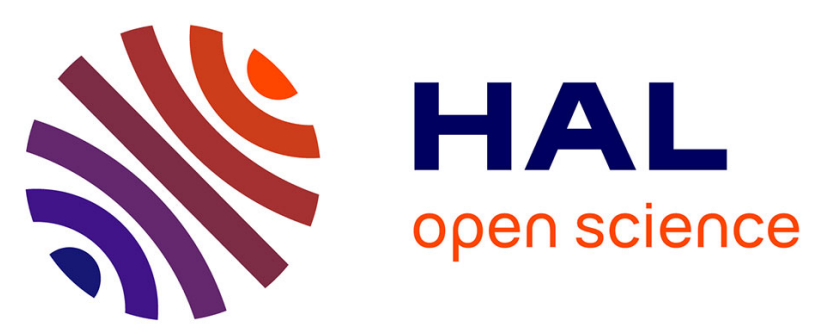

\title{
Les Legal Consciousness Studies comme laboratoire d'un régime renouvelé de connaissance sur le droit Présentation du dossier
}

\author{
Jacques Commaille, Stéphanie Lacour
}

\section{- To cite this version: \\ Jacques Commaille, Stéphanie Lacour. Les Legal Consciousness Studies comme laboratoire d'un régime renouvelé de connaissance sur le droit Présentation du dossier. Droit et Société, 2018, 100, pp.547-558. 10.3917/drs1.100.0547 . halshs-03327378}

\section{HAL Id: halshs-03327378 \\ https://shs.hal.science/halshs-03327378}

Submitted on 27 Aug 2021

HAL is a multi-disciplinary open access archive for the deposit and dissemination of scientific research documents, whether they are published or not. The documents may come from teaching and research institutions in France or abroad, or from public or private research centers.
L'archive ouverte pluridisciplinaire HAL, est destinée au dépôt et à la diffusion de documents scientifiques de niveau recherche, publiés ou non, émanant des établissements d'enseignement et de recherche français ou étrangers, des laboratoires publics ou privés. 


\title{
Les Legal Consciousness Studies comme laboratoire d'un régime renouvelé de connaissance sur le droit
}

\author{
Présentation du dossier*
}

Jacques Commaille et Stéphanie Lacour

Les Legal Consciousness Studies ${ }^{l}$ sont un courant de recherche qui occupe une place importante dans 1'histoire contemporaine des Socio-Legal Studies ${ }^{2}$ aux Etats-Unis. Nous ne saurions avoir la prétention de le faire découvrir aux lecteurs de Droit et Société. Outre plusieurs articles déjà consacrés à ce courant dans la revue ${ }^{3}$, ou dans d'autres publications ${ }^{4}$, la littérature européenne est riche d'analyses, de commentaires qui lui sont consacrés ou lui font référence ${ }^{5}$. Plus spécifiquement, le grand intérêt dont il a fait l'objet en France s'inscrit dans une tradition d'échanges existant depuis plusieurs décennies entre des sociologues du droit et

\footnotetext{
*Nous tenons à remercier tout particulièrement Emilie Cloâtre et Olivier Leclerc pour les remarques et commentaires qu'ils ont formulés sur les premières versions de tous les articles de ce dossier, qui nous ont été très précieux.

${ }^{1}$ Nous employons ici le concept de Legal Consciousness Studies dans le prolongement de la manière dont ce mouvement a été, depuis le milieu des années 2000, présenté en France par des sociologues du droit. Nous ne cherchons pas à suggérer que les études de Legal Consciousness revêtent, aux Etats-Unis, la même portée épistémique que des mouvements plus institutionnalisés, tels que les Gender Studies ou encore les Science and Technology Studies, dont il sera question plus loin (A cet égard, V. Lucas Monteil, Alice Romerio, «Des disciplines aux «studies ».Savoirs, trajectoires, politiques », Revue d'anthropologie des connaissances, 2017/3 (Vol. 11, n³), pp. 231-244). Pour autant, la constitution interdisciplinaire de ce mouvement, tout comme sa dimension profondément critique, son ancienneté et le positionnement stratégique de ses membres au sein de la Law and Society Association, nous paraissent plaider pour l'octroi d'un tel label. L'article qui ouvre ce dossier «After Legal Consciousness... » nous semble, à cet égard, témoigner d'une volonté affirmée de Susan Silbey, dans le tout premier numéro de l'Annual Review of Law and Social Sciences paru en 2005, de réaffirmer la pertinence et les cadres analytiques d'un mouvement appelé à perdurer dans le paysage international.

2 Comme le souligne Susan Silbey dans l'article qui ouvre ce dossier, le terme "Socio-legal Studies", initialement plus usité en Europe, est aujourd'hui devenu commun également aux Etats Unis. Nous l'utilisons, à l'image de cette auteure, de manière interchangeable avec «Law and Society movement », en anglais comme en français.

3 V. Mauricio Garcia Villegas, "Symbolic Power Without Symbolic Violence ? Critical Comments on Legal Consciousness Studies in USA", Droit et Société, 2003/1, n53, pp. 137-163 ou encore Jérôme Pélisse, « Consciences du temps et consciences du droit chez des salariés à 35 heures », Droit et Société, 2003/1, n53, pp. 163-186.

${ }^{4}$ Voir, par exemple : Jérôme Pélisse, «A-t-on conscience du droit ? Autour des Legal Consciousness Studies », Genèses 2005/2, , n 59, pp. 114-130 ; Jérôme Pélisse, «Les Legal Consciousness Studies : une sociologisation domestiquée des Critical Legal Studies ?», dans Hourya Bentouhami, Ninon. Grangé Anne Kupiec, Julie Saada (dir.), Le souci du droit. Où en est la théorie critique?, Paris, Sens \& Tonka\&Cie, coll. « Théorie critique », 2010, pp. 1-15.

${ }^{5}$ Nous pensons en particulier à un article témoignant de l'intérêt croissant porté en Europe au courant des Legal Consciousness Studies: Marc Hertogh, "A 'European' Conception of Legal Consciousness: Rediscovering Eugen Ehrlich”, Journal of Law and Society, vol. 31, n 4, December 2004, pp. 457-481.
} 
des membres de la Law and Society Association, notamment avec Susan Silbey, qui en est l'une des grandes figures et dont l'œuvre est au centre du projet que restitue le présent dossier.

\section{Les Legal Consciousness Studies pour mettre la recherche sur le droit à l'épreuve}

Il ne s'agissait donc pas pour nous de faire connaître ce courant de recherche. C'est déjà fait, et superbement fait ainsi qu'en témoigne l'article publié dans ce même dossier de la revue par Vincent-Arnaud Chappe, Anna Egea et Jérôme Pélisse sur la réception en France des travaux de Susan Silbey. Dans cet article, les auteurs se livrent de façon méthodique à une observation des formes et de l'ampleur de l'intérêt porté à ces travaux par la recherche française notamment la sociologie, ceci depuis de nombreuses années. Une démarche différente s'est imposée progressivement à nous. Elle se situe dans le prolongement de cet intérêt initialement porté par des sociologues français soucieux de se démarquer d'un contexte où la sociologie du droit était avant tout une sociologie du droit de l'État. Notre propre démarche s'est développée à partir de la conviction que ce courant, et plus précisément l'œuvre d'une de ses plus éminentes représentantes, pouvait se voir conférer le statut d'épreuve ${ }^{6}$ pour notre collectif à de multiples titres.

Cette épreuve devait tout d'abord permettre, de façon tout à fait originale, de tirer parti de la diversité assumée de la composition d'un groupe de réflexion qui s'est réuni pendant plus de dix-huit mois et dont les membres venaient de disciplines différentes (droit, sociologie, science politique, ethnographie inspirée de la sociologie et de l'anthropologie...) mais aussi de spécialisations différentes (par exemple, dans le domaine des rapports entre droit, sciences et techniques, dans celui du droit de la culture et du patrimoine, de la sociologie du droit ou de la sociologie des sciences et des techniques...) $)^{7}$. Comment faire

\footnotetext{
${ }^{6}$ Nous utilisons ici le mot «épreuve » dans le sens d'expérimentation d'une mise en commun de compétences diverses sur notre objet. Il ne s'agit donc pas d'une référence à l'usage qui est fait du mot dans la « sociologie pragmatique » comme «sociologie des épreuves», ceci bien que cette sociologie ait pu être une source d'inspiration parmi d'autres dans nos analyses.

${ }^{7}$ Ont participé aux réunions mensuelles de ce que nous avons, par commodité, dénommé le « Séminaire Silbey » non seulement Jacques Commaille (sociologue, ISP) et Stéphanie Lacour (juriste, ISP) mais également Virginie Albe (didactique des sciences, ISP), Brigitte Frotiée (sociologue, ISP), Cléa Hance (juriste, ISP), Nicole Kerchen (juriste, ISP), Isabelle de Lamberterie (juriste, ISP) Daniela Piana (politiste, Université de Bologne), Emilia Schijman (anthropologue-sociologue, Centre Maurice Halbwachs), Diana Villegas (juriste, Université PanthéonAssas-Paris II), Noé Wagener (juriste, Université de Rouen) et Anne Wyvekens (juriste-sociologue, ISP). Le travail au long cours accompli au sein de ce groupe a donné lieu à une journée de workshop dans les locaux de l'Institut d'Etudes Avancées de Paris, le 9 juin 2017, au cours de laquelle nous avons pu ouvrir nos réflexions aux apports de Mauricio Garcia Villegas (juriste et sociologue, Université nationale de Colombie, Université de Madison), Liora Israël (sociologue, EHESS, Centre Maurice Halbwachs), Jérôme Pélisse (sociologue, Centre de sociologie des Organisations, Sciences Po Paris), Vincent-Arnaud Chappe (sociologue, Centre de Sociologie de
} 
naître, de ces différentes compétences et centres d'intérêt, une convergence de regards sur le droit et amorcer les prémices d'une interdisciplinarité dont nous avions une vive conscience qu'elle ne constituait pas un enjeu seulement pour notre groupe mais pour toute la recherche sur le droit ? Le courant Legal Consciousness nous offrait l'opportunité de nous rassembler et d'entreprendre la construction d'un édifice commun ayant vocation à être partagé par la recherche sur le droit en général. Il autorisait également, entre les membres de notre collectif, nombre de discussions inédites sur certains aspects fondateurs d'une connaissance spécifique du droit.

Ce courant s'inscrivait en effet dans des pratiques et visions de la production de connaissances sur le droit qui demeurent, en France, en rupture par rapport aux approches dominantes. La perspective au fondement de ce courant est à l'opposé de ce qui a longtemps caractérisé - aux yeux des juristes ${ }^{8}$ comme des sociologues $^{9}$ eux-mêmes - la sociologie du droit française, une sociologie fortement dépendante de l'univers juridique et conçue, à ce titre, comme une ingénierie sociale au service de l'amélioration du droit, droit de l'Etat à titre quasi exclusif.

Parallèlement, les chercheurs juristes ont très vite perçu la fonction heuristique susceptible d'être remplie par ce courant. Il justifie en effet leur aspiration à dépasser la production de connaissances de type dogmatico-doctrinal répondant aux canons répandus du formalisme juridique. Cette approche les incite à rechercher, au-delà des textes, des

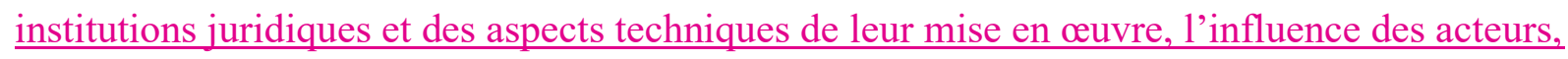
leurs stratégies, leurs valeurs et intérêts. Elle ouvre leur horizon à l'examen de l'économie des $\underline{\text { relations avec d'autres normativités, de l'influence de contextes institutionnels, culturels, }}$ économiques, politiques et sociaux, plus largement, d'autres formes de régulation Parallèlement, les chercheurs juristes ont très vite perçu la fonction heuristique susceptible d'être remplie par ce courant, justifiant leur aspiration à dépasser la production de

l'Innovation, Ecole des Mines), Julie Ringelheim (juriste, Université Catholique de Louvain) et Jean De Munck (sociologue et juriste, Université Catholique de Louvain).

${ }^{8}$ Voir, par exemple, en référence à sa « sociologie législative », Jean Carbonnier, Sociologie juridique, Paris, PUF, 1978.

${ }^{9}$ Jacques Commaille, «Sociologie juridique », in Denis Alland et Stéphane Rials, Dictionnaire de la culture juridique, Paris, Lamy-PUF, 2003, pp. 1423-1427. Une telle orientation ne s'explique pas seulement par une dépendance par rapport aux juristes. Elle peut résulter également d'une tradition suivant laquelle elle correspondrait à «l'une des conceptions les plus influentes du droit en sciences sociales » : Brian Z. Tamahana, "An Analytical Map of Social Scientific Approaches to the Concept of Law", Oxford Journal of Legal Studies, 1995, vol.15, No 4, Winter, pp.501-53, article traduit par Baudouin Dupret, disponible en ligne sur Clio@Themis, 2009,numéro 1. 
connaissances de type dogmatico-doctrinat ${ }^{10}$ répondant aux canons répandus du formalisme juridique, l'étude des textes et des institutions juridiques, des aspects techniques de leur mise en @uvre, pour rechercher l'influence des acteurs, leurs stratégies inspirées par la poursuite d'intérêts comme de valeurs, pour prendre en compte l'économie des relations avec d'autres normativités, l'influence de contextes institutionnels, culturels, économiques, politiques et sociaux, plus largement, d'autres formes de régulation.

Les chercheurs relevant du domaine «Science and Technology Studies » (STS), enfin, se sont saisis de cet objet pour mettre en valeur des analogies entre les approches sur le droit et celles relevant des études des sciences et des techniques. Ce qui se dégageait, c'était le même constat d'un processus de désacralisation des sujets étudiés, «le Droit» et «la Science ». Face à l'institution a priori d'une «Vérité » de l'ordre du « déjà-là » ou à établir, la recherche démontrait l'évidence commune d'une construction sociale dans laquelle interfèrent de multiples facteurs extérieurs à ce qui est parfois présenté comme une dynamique intrinsèque de la connaissance. C'est ainsi que, de façon analogue à la déconstruction par les «Legal Consciousness Studies» de la représentation d'un droit monolithique et transcendant, les chercheurs des «Science and Technology Studies» ont, dans des espaces de temps comparables, mis en évidence l'extrême diversité des pratiques de sciences, leur caractère situé et contingent, contribuant ainsi à rompre avec la représentation d'une « essence de la science $»^{11}$.

\section{Quatre ruptures dans le régime de connaissance sur le droit}

Loin d'une simple manifestation inattendue de la sérendipité dans le champ des sciences sociales, cette dynamique de production de connaissances, cette optimisation des différences en vue d'établir des convergences, n'a été possible que grâce à une interaction permanente avec l'objet choisi : l'œuvre de Susan Silbey, représentante éminente du courant des Legal Consciousness Studies. Cette œuvre nous est progressivement apparue comme recélant des incitations pour inscrire nos propres travaux dans un nouveau régime de production des connaissances. Nous avons été sensibles à ce qu'elle préconisait ou autorisait de ruptures avec certains des schémas consacrés de la connaissance sur le droit.

\footnotetext{
${ }^{10} \mathrm{~V}$. Antoine Jeammaud, «Une typologie des activités savantes prenant le droit pour objet », in Sciences de l'homme et de la société, CNRS, 1999, n54. p. 15 ou encore, du même auteur, « La part de la recherche dans l'enseignement du droit », Jurisprudence. Revue Critique, n 1 1, 2010, pp. 181-213.

${ }^{11}$ Dominique Pestre, Introduction aux Science Studies, Paris, La Découverte, coll. « Repères », 2006, p. 6.
} 
Une de ces ruptures est illustrée par ce que l'on peut considérer, à la lecture du livre de référence de ce mouvement ${ }^{12}$, comme un élément d'une révolution paradigmatique dans la recherche sur le droit. Il s'agit rien moins que de rompre avec une vision causale de la relation entre Droit et Société. Cette relation causale conduisait non seulement à envisager les deux termes comme relevant d'espaces distincts, potentiellement autonomes, mais encore à poser la question de leurs relations en termes d'effectivité ou de formes diverses d'influences réciproques entre le juridique et le social. A cette conception est substituée ici celle suivant laquelle «les phénomènes légaux [sont] des éléments constitutifs de la réalité sociale plutôt que (...) des éléments enserrés dans des appareils institutionnels destinés à intervenir sur cette réalité $»^{13}$. Dans cette perspective, le droit ne peut plus être étudié comme un phénomène séparé de la société et il devient indispensable de se détourner « de l'étude des rapports entre droit et société vers celle du droit en société ${ }^{14}$. Ce constat était déjà esquissé par Stuart Scheingold ${ }^{15}$ au travers de l'idée de « mythe du droit ». Les tenants du paradigme constitutif ${ }^{16}$ sont ensuite venus le préciser. Les analyses portant sur les usages sociaux du droit et les «consciences du droit» que ces études révèlent vont toutefois apporter un degré de raffinement supplémentaire à cette perspective. Elles justifient en effetune nouvelle approche de la légalité définie, comme le rappelle avec force Susan Silbey, comme « une forme de relations et de représentations sociales à qui est donnée quotidiennement force de loi à travers des schèmes que les gens invoquent pour construire le sens de leurs actions et de celles des autres $»^{17}$.

Une possibilité nouvelle découle de cette première rupture. Le droit, la vie du droit peut être approchée, non pas uniquement au travers d'une étude surplombante, celle d'un droit qui s’imposerait de facto à la société et à ses membres, mais également à partir de ce

\footnotetext{
${ }^{12}$ Patricia Ewick et Susan Silbey, The Common Place of Law. Stories from Everyday Life, Chicago, The University of Chicago Press, 1998.

13 Jérôme Pélisse, «Conscience du temps et consciences du droit chez les salariés à 35 heures », p. 164.

${ }^{14}$ Susan S. Silbey, “After legal consciousness”, Annual Review of Law and Social Science, 2005, vol. 1, pp. 323368. Voir la traduction du texte dans ce numéro, p. 9 (avant mise en page dans la revue).

${ }^{15}$ Stuart A. Scheingold, The Politics of Rights: Lawyers, Public Policy, and Political Change, New Haven, 1974, Yale University Press.

${ }^{16}$ V. notamment : Alan Hunt, Explorations in Law and Society. Toward a Constitutive Theory of Law, New York, London, Routledge Editions, 1993 ; voir également : Sally Engle Merry, Getting Justice and Getting Even. Legal Consciousness among Working Class, Chicago, University of Chicago Press, 1990 ; .Michael McCann, "On Legal Rights Consciousness: A Challenging Analytical Tradition", préface de l'ouvrage dirigé par Benjamin Fleury-Steiner et Laura Beth Nielsen, The New Civil Rights Research : A Constitutive Approach », Ashgate Publishing Limited, June 2006.

${ }^{17}$ Patricia Ewick et Susan Silbey, op. cit., p. 31-32.
} 
que les citoyens font, pensent, voire, dans certains cas, ignorent du droit. Nous verrons, dans le contenu même du dossier, combien une telle vision, à l'opposé d'une approche top down, offre d'opportunités de revenir et réétudier à nouveaux frais des traditions de la pensée juridique ou sur le juridique qui ont été constamment marginalisées : celle du droit vivant ${ }^{18}$ comme celle du droit social ${ }^{19}$ de Georges Gurvitch quand il relativise le «monisme juridique étatique » pour porter de l'attention à un droit « connecté à la société », «immergé dans la société $»^{20}$.

Ce choix d'inverser en quelque sorte le regard sur le droit rompt enfin avec des méthodes de recherche éprouvées par le passé. Il porte en germe l'exigence de formes nouvelles de l'empirie dans la démarche de recherche. Confronter l'observation du droit à ce qu'il devient dans la société oblige en effet à plusieurs pas de côté. Il s'agit d'abord de sortir non seulement du formalisme juridique classique, des approches purement rhétoriques sur le sens du droit, les procédures de son élaboration et de sa mise en œuvre. Il faut aussi, comme le rappelle Susan Silbey, se démarquer tout à la fois des méthodes d'enquête très globalisantes «portant sur les attitudes, les connaissances et l'usage du droit par les citoyens » et des «méthodes ethnographiques d'observations extensives et d'entretiens intensifs afin d'étudier les litiges, les comportements conflictuels et le recours au droit $»^{21}$ qui avaient, jusqu'alors, mobilisé les sociologues du droit américains et n'observaient pas la légalité dans la vie quotidienne mais seulement dans les manifestations pathologiques du droit.

Comment appréhender la complexité des formes d'appropriations matérielles mais aussi mentales du droit dans les multiples façons dont les individus pensent et se pensent dans la société, dans l'immense diversité des pratiques dont le droit va faire l'objet dans des situations différentes, inspirées par des dispositions sociales et culturelles, des stratégies, des situations, etc. ? Nous verrons qu'il y a là un enjeu considérable dans le choix des méthodes et que, peut-être, les quelque 430 entretiens réalisés par Patricia Ewick et Susan Silbey ${ }^{22}$ pour

\footnotetext{
${ }^{18}$ E. Ehrlich, The Fundamental Principles of the Sociology of Law, 1975, New York, Russell \& Russell, cité par Brian Z.. Tamahana, «An Analytical Map of Social Scientific Approaches to the Concept of Law », op. cit.

${ }^{19}$ Noé Wagener, « Droit social », dans Marie Cornu, Fabienne Orsi et Judith Rochfeld (dir.), Dictionnaire des biens communs Paris, PUF, collection «Quadrige », 2017, pp. 433-439.

${ }^{20}$ Jean-Guy Belley, «Georges Gurvitch et les professionnels de la pensée juridique », Droit et Société, 1986, n ${ }^{\circ}$, pp. 353-370; du même auteur: «Le 'droit social' de Gurvitch : trop beau pour être vrai ? ", Droit et société, 2014/3 ( $\left.n^{\circ} 88\right)$, pp. 731-746.

${ }^{21}$ Susan S. Silbey, «After Legal Consciousness », op. cit., spécialement pp. 20-29. (REVOIR PAGINATION SUR LES EPREUVES) ${ }^{\circ}$

${ }^{22}$ Patricia Ewick et Susan Silbey, op. cit.
} 
leur recherche, malgré l'ampleur de l'investissement qu'un tel chiffre représente, ne peuvent totalement suffire à épuiser le sens de la réalité complexe de la vie du droit qui est aussi faite des « compétences juridiques des acteurs sociaux », « des stratégies manipulatrices des sujets sociaux $»^{23}$ et de mobilisations collectives.

Très logiquement, ces trois étapes introduisent une dernière rupture. Tout comme le dévoilement de la réalité de « la Science » implique la remise en cause du monopole du regard de ceux qui la font sur ce qu'ils font, une rupture avec l'idée d'un possible monopole de la connaissance sur le droit s'impose d'évidence. Bien entendu, il n'est pas question d'une sorte d'indifférenciation des compétences mais bien d'une complémentarité qui aille au-delà du partage usuellement pratiqué entre approches internes et externes du droit ${ }^{24}$ et permette l'édification d'une troisième voie. Celle-ci fonctionne d'autant plus que le souci d'affirmation constante, comme un préalable, d'identités disciplinaires propres s'efface au profit de la poursuite d'un objectif commun de production de connaissances originales. C'est ainsi que, conscients de la fonction heuristique de la démarche de Susan Silbey, il nous est apparu très vite que cela n'avait aucun sens de s'interroger, comme c'est si souvent le cas en France ${ }^{25}$, sur le fait de savoir si elle était juriste, sociologue ou politiste et, de la même façon, de nous demander, de façon obsessionnelle, depuis quels territoires de la connaissance nous intervenions dans les riches débats qui réunissaient notre groupe.

Le choix de travailler sur un tel objet - l'œuvre de Susan Silbey pour ce qu'elle nous dit de la conscience du droit - avait bien pour objectif d'éprouver certains des attributs d'un régime de production des connaissances auquel nous n'étions pas totalement rompus, notamment sur le plan épistémologique.

\footnotetext{
${ }^{23}$ Simona Cerutti, « Normes et pratiques, ou de la légitimité de leur opposition », dans Bernard Lepetit (dir.), Les formes de l'expérience. Une autre histoire sociale, Paris, Albin Michel, 1995, pp. 130, 131.

${ }^{24}$ Sur ce débat «Approche interne-approche externe», voir notamment: François Ost et Michel van de Kerchove, «De la scène au balcon. D’où vient la science du droit? », dans François Chazel et Jacques Commaille (dir.), Normes juridiques et régulation sociale, Paris LGDJ, coll. « Droit et Société, pp. 67-80 ; Pierre Bourdieu, « Les juristes, gardiens de l'hypocrisie collective », dans François Chazel et Jacques Commaille (dir.), Normes juridiques et régulation sociale, op. cit., pp. 95-99; André-Jean Arnaud, « La valeur heuristique de la distinction interne/externe comme grande dichotomie pour la connaissance du droit: éléments d'une démystification », Droit et Société, 1986/2, pp. 139-141 et plus récemment Evelyne Serverin, « Points de vue sur le droit et processus de production des connaissances », R.I.E.J., 2007, n 59, pp. 73-91.

${ }^{25}$ Et pas uniquement dans le champ des études sociojuridiques, puisque, comme le relève Olivier Leclerc, c'est une question que l'on a aussi beaucoup posée au sujet des travaux de Sheila Jasanoff dans celui des STS. En ce sens, v. «Le droit et la science en action. Sheila Jasanoff traduite et présentée par Olivier Leclerc », Dalloz, Rivages du droit, 2013, spécialement. p. 10.
} 
Il nous reste à présenter les diverses facettes d'une opérationnalisation de cette épreuve.

\section{L'économie générale du dossier}

Il nous a semblé d'abord utile de proposer un travail de traduction d'un article de Susan Silbey : «After Legal Consciousness...». Cet article, qui ouvre notre dossier, est à la fois une synthèse des grandes orientations de ce courant mais également un témoignage des réactions de Susan Silbey à la réception dont ces travaux ont fait l'objet dans l'univers nordaméricain. Il est également une entreprise de recadrage du mouvement qu'elle entendait lancer, de même qu'un témoignage sociologiquement intéressant d'une actrice importante de la recherche américaine contemporaine sur le droit, auteure d'une œuvre, participante en vue dans des collectifs ainsi que le montrent nombre de publications co-signées, animatrice de recherche notamment à partir de sondu statut_qu'elle a eu de directrice du Département d'anthropologie au $\mathrm{MIT}^{26}$. Ce travail de traduction a accompagné notre démarche d'analyse critique. La traduction a été réalisée en partie dans le cadre d'un atelier de traduction du département de sciences sociales de l'Ecole normale supérieure de Paris-Saclay, par des élèves de cette institution ${ }^{27}$, sous la supervision de deux enseignantes-chercheures, Caroline Vincensini et Laure de Verdalle, assistées des membres du séminaire ${ }^{28}$. Ce travail de traduction a fait suite à celui, précurseur, réalisé quelques années auparavant dans les mêmes conditions $^{29}$. Bien entendu, ce travail a conduit classiquement à soulever de nombreuses réflexions autour de la difficulté de transposer certains concepts ou manières de penser, et plus généralement de la difficulté de l'importation d'analyses inspirées par une culture et des visions du monde différentes entre le pays émetteur et le pays récepteur, difficulté que Liora

\footnotetext{
${ }^{26}$ Nous n'ignorons pas que derrière une œuvre, il y a une personne avec son histoire personnelle, ses inclinations éthiques ou politiques, ses stratégies, etc. C'est une dimension qui mériterait particulièrement d'être approfondie, ce que nous souhaitions faire si les circonstances ne nous avaient pas empêchés d'avoir plus d'interactions avec l'auteure. Il reste que la réponse de Susan Silbey à nos analyses-, qui conclut le présent dossier, offre à cet égard des éléments d'information du plus grand intérêt.

${ }^{27}$ Ont ainsi contribué à cette traduction, sous la direction de leurs enseignants, Eloïse Alluyn-Fride, Augustin Ayoub, Paul Babin, Sofiane Bouarif, Valentin Barbet, David Colla, Adrian Jean-Baptiste, Alice Leuleu, Benjamin Péron, Clément Ramos, Ruby Schultz et Constant Thiard.

${ }^{28}$ Brigitte Frotiée, Isabelle de Lamberterie, Cléa Hance, Stéphanie Lacour, Jacques Commaille, Anne Wyvekens, Virginie Albe, Emilia Schijman, Daniela Piana, Noé Wagener, ont participé à cette traduction dans le cadre de leur séminaire d'études.

${ }^{29} \mathrm{Il}$ s'agit d'un travail portant sur un chapitre de l'ouvrage de Patricia Ewick et Susan Silbey, réalisé à l'ENS de Cachan, sous la responsabilité de Liora Israël et Jérôme Pélisse. Voir leur article : «Quelques éléments sur les conditions d'une 'importation' (Note liminaire à la traduction du texte de Susan Silbey et Patricia Ewick)», Terrains et Travaux, $\mathrm{n}^{\circ}$ 6, 1/2004, pp. 101-111.
} 
Israël et Jérôme Pélisse avaient justement renvoyée à «l'épistémologie même des sciences sociales. Celles-ci ne sont-elles pas indissociablement des produits et des analyses des sociétés dont elles proviennent et qu'elles étudient ? $»^{30}$.

Notre travail s'est ensuite déployé sur une longue durée, ce qui aurait pu constituer une gêne si l'assistance technique dont nous avons bénéficié tout au long de ce parcours de recherche collectif n'avait pas permis la mise en commun, en ligne, de l'ensemble des ressources que nous explorions - lesquelles ne se sont pas résumées, cela va de soi, aux articles publiés par Susan Silbey -, de nos fiches de lectures, et de comptes rendus très complets de l'ensemble de nos échanges. L'appropriation réciproque de vocabulaires et concepts initialement distincts tout comme de méthodes et approches intellectuelles variées est un réel enjeu de la recherche interdisciplinaire, elle est en effet indispensable à la construction d'un objet commun. Ces atouts organisationnels et théoriques y ont amplement contribué et méritent, à ce titre, d'être soulignés.

Les quatre contributions qui suivent étaient initialement rédigées sous la forme de questions adressées à Susan Silbey, que nous avions prévu de rencontrer à Paris pour quelques séances de travail communes. Susan Silbey revient sur les raisons qui ont, jusqu'à présent, transformé ces rendez-vous en échanges épistolaires. Son texte, disponible dans ce dossier en anglais, a fait l'objet d'une traduction, publiée en ligne sur le blog de la revue Droit \& Société $^{31}$, par Cléa Hance avec la collaboration de Stéphanie Lacour et Anne Wyvekens.

Nous présentons ici ces textes comme autant d'interrogations interdisciplinaires nées de la richesse de l'œuvre de Susan Silbey. En nous penchant sur l'histoire, les méthodes, les choix épistémologiques et les terrains de l'auteure, au cœur des Legal Consciousness Studies, nous avons mis nos connaissances à l'épreuve d'une littérature américaine engagée et originale, dont l'audace a légitimement suscité, chez chacun des auteurs de ce dossier, l'envie de discuter, de partager et de se projeter collectivement dans un nouveau régime interdisciplinaire de production de connaissances sur le droit.

Le texte rédigé par Anne Wyvekens, tout d'abord, resitue cette œuvre dans l'histoire sociale des études socio-juridiques américaines, qu'il s'agisse de ses formes institutionnelles (la Law and Society Association, le Amherst Seminar on Legal Ideology and Legal Process), ou de ses courants de recherche (depuis la Sociological Jurisprudence et le Legal Realism en

\footnotetext{
${ }^{30}$ Ibid., p. 111.

${ }^{31}$ Indiquer l'adresse de la traduction.
} 
passant par les Critical Legal Studies). Elle montre à la fois l'audience et les critiques dont le mouvement des LCS a pu faire l'objet, les réflexions sociologiques qu'il a suscitées quant à la place et au statut de ses auteurs (du côté des « dominés ») et à leur rapport au militantisme. Ouvrant le dialogue avec l'auteure, Anne Wyvekens interroge les perspectives de recherche que l'œuvre de cette dernière fait émerger, de même que le contexte dans lequel cette œuvre se déploie et qu'elle cherche, de fait, à innerver, à la fois dans l'univers académique et dans le champ politique étatsunien. Comme observatrice et actrice, Susan Silbey se place au cœur de l'évolution moderne des études sociojuridiques. Ce retour sur l'histoire des LCS permet assurément d'en mesurer la modernité.

Dans la contribution rédigée conjointement par Daniela Piana, Emilia Schijman et Noé Wagener, c'est la question des positions épistémologiques et méthodologiques de l'auteure qui est approfondie au regard des réflexions qu'elles ont fait naitre chez les auteurs. Dans l'alternative classique entre les « sources juridiques des pratiques sociales » et « les « sources sociales des règles juridiques », les LCS ont, leur semble-t-il, privilégié une approche qui valorise malgré tout «le Droit» institué comme étant celui qui est «incorporé » dans la conscience des citoyens ordinaires interrogés. Le «droit vivant» d'Eugen Ehrlich ou le «droit social » de Georges Gurvitch constituent, à cet égard, des approches différentes. Pour les auteurs, ce choix est indissociable d'options méthodologiques dont ils démontrent les limites. Une approche ethnographique, plus que l'interview, aurait en effet permis d'inscrire les représentations du droit des acteurs sociaux concernés dans des contextes, suivant des dispositions sociales, des «champs» d'action, etc. Elle aurait également pu contribuer à démontrer en quoi ces acteurs sociaux sont aussi susceptibles d'être des acteurs du droit, d'agir sur le droit, d'en être une des sources possible. Loin de s'en tenir à ces regrets, les auteurs soulignent surtout combien l'approche des LCS ouvre des perspectives plus générales pour les savoirs de sciences sociales sur le droit et les normativités, en réinterrogeant à nouveaux frais l'économie des échanges entre micro et macro et leur articulation dans le cadre d'études bien outillées de sciences sociales sur l'Etat de droit.

Virginie Albe et Stéphanie Lacour centrent leur réflexion sur une mise en perspective $\mathrm{du}$ courant Legal Consciousness par rapport aux analyses relevant des «Science and Technology Studies» (STS). Susan Silbey consacre en effet depuis plusieurs années des recherches aux manifestations du droit dans les laboratoires de recherche. Tirant parti de la complémentarité de leurs approches disciplinaires tout comme des parallèles existant entre les 
conditions d'émergence, les options théoriques et méthodologiques et les racines idéologiques de ces deux mouvements, elles questionnent le rendez-vous manqué entre LCS et STS. Comme ceux des autres auteurs de ce dossier, leur point de vue est toutefois plus prospectif que nostalgique. Rapprocher ces deux mouvements de recherche permettrait en effet, selon elles, de mieux étudier les interactions entre droits, sciences et techniques. En tirant parti des réussites et des échecs - relatifs - des deux mouvements, il deviendrait ainsi possible de dessiner la voie d'une approche réellement critique des normativités à l'œuvre dans la production de connaissances et les artéfacts scientifiques et techniques, une approche qui prenne au sérieux non seulement les communautés et les lieux dans lesquels ils se déploient mais aussi les normes qu'ils contiennent et qui, parfois, les contraignent.

Jacques Commaille, enfin, fait le choix de se situer pleinement dans le registre de la controverse. Il reprend le constat fait par ailleurs d'une dissonance entre les études empiriques et le cadre théorique ${ }^{32}$ mobilisé par les LCS. À la lecture des observations de Susan Silbey tirées des entretiens réalisés, laissant notamment entrevoir des capacités de résistance de la part des citoyens ordinaires, il s'interroge sur la formulation d'un constat général qui reste celui d'une «legal hegemony » qui s'imposerait massivement. Un tel positionnement conduit alors Susan Silbey à solliciter des théories européennes valorisant exclusivement cette idée d'hégémonie et de systèmes de domination. Ces théories sont logiquement associées à une adhésion à la théorie de la «fausse conscience», dans la mesure où l'hégémonie et la domination «ne sont pas seulement tolérées mais suscitent l'adhésion des populations dominées $»^{33}$. La conséquence est que ne sont pas véritablement prises en compte d'autres théories fondées sur la thèse précisément d'une capacité de résistance, d'appropriation du droit comme mode de résistance ou comme support d'engagement dans un conflit.

Susan Silbey a bien voulu répondre longuement, le dossier se termine par cette réponse circonstanciée à ces quatre notes de commentaires et de questionnements critiques, ainsi, plus généralement, qu'à cette entreprise d'analyse critique. Après être revenue sur son positionnement de recherche ayant le droit comme objet, se livrant à un travail de définition de cet objet et considérant qu'il relevait d'abord d'une approche culturelle, Susan Silbey se penche sur sa définition de la « légalité », celle-ci étant inspirée par une référence privilégiée aux acteurs sociaux. La suite de son riche commentaire représente un exceptionnel travail de

\footnotetext{
${ }^{32}$ Mauricio Garcia Villegas, “On Pierre Bourdieu's Legal Thought”, Droit et Société, n 56-57, 1/2004, p. $57-70$.

${ }^{33}$ Susan S. Silbey, “After Legal Consciousness”, op. cit. p. 328.
} 
réflexivité de la chercheure sur son parcours intellectuel et son œuvre. Elle y resitue - en justifiant leur place spécifique - les Legal Consciousness Studies dans une histoire contemporaine des travaux de la Law and Society Association aux Etats-Unis. Prenant acte de l'influence sur l'œuvre que le chercheur produit de sa condition de sujet social, elle inscrit ses propres travaux dans une trajectoire personnelle et professionnelle qu'elle décrit longuement et au regard des influences, professionnelles comme amicales, dont cette trajectoire a pu s'enrichir au fil des ans.

Ce travail de réflexivité se prolonge dans les réponses faites aux questions posées dans les notes évoquées supra, dans la mesure où l'auteure se réfère par exemple à ses recherches sur la place du droit dans les laboratoires, rappelant au passage que le droit comme la science sont des institutions sociales. Face aux interrogations sur l'adéquation entre ses données empiriques et les théories qu'elle mobilise, elle mentionne notamment ce qui peut tenir dans cette discussion aux spécificités culturelles de la société américaine par rapport aux sociétés européennes. Son engagement sans réserve dans cette dynamique de la discussion l'entraîne à réaffirmer avec force la nécessité de construire une véritable théorie de la légalité, d'une légalité saisie comme «terrain de pouvoir », une théorie nourrie de la vive conscience de l'existence dans ce domaine de contradictions fondamentales dont l'analyse même est susceptible de leur donner une fonction heuristique. Enfin, comment ne pas être sensible au fait que le développement d'une telle pensée, dont il nous est offert ici un superbe témoignage, est traversé par le souci constant de concourir à la réalisation d'un idéal démocratique ${ }^{34}$, justifiant cette volonté farouche d'emprunter des voies nouvelles pour contribuer au dévoilement du droit.

\section{Des potentialités d'un régime de connaissance renouvelé}

Le généreux engagement de Susan Silbey a donné tout son sens à notre projet de faire des Legal Consciousness Studies, et particulièrement de l'analyse de l'œuvre de cette collègue, le point de départ de nos réflexions. Il a consacré son statut d'épreuve en vue de faire de nos différences un atout pour penser un régime de production de connaissances

\footnotetext{
${ }^{34}$ En étant particulièrement sensible, dans ses orientations de recherche mais aussi au-delà, à la question de l'égalité : celle en général entre les citoyens ; celle dont sont privées les femmes, question pour laquelle elle s'est notamment engagée dans la défense du statut de ses étudiantes et collègues féminines dans le milieu universitaire américain.
} 
renouvelé sur le droit. De cette stimulante expérience, nous tirons finalement deux enseignements forts. C'est du dialogue, tout d'abord, que nous nous donnons les moyens d'entretenir avec d'autres recherches prenant le droit comme objet, plus que d'une forme de déférence, que peut découler l'enrichissement de nos travaux. Cet objet, le droit, par ailleurs, offre aux sciences sociales l'opportunité de mobiliser de nouveaux savoirs de manière heuristique sur l'ensemble des transformations de nos sociétés.

Le premier enseignement concerne le sentiment d'un déplacement dans le positionnement souvent adopté face à la production américaine : celui consistant à la placer au sommet de l'excellence dans le cadre d'une hiérarchie implicite de la recherche sur le droit, la réussite dans l'espace européen, en termes de notoriété ou en termes... de carrière, passant alors simplement par le fait de fournir la preuve d'une connaissance érudite de cette production et de la fréquentation de ses auteurs. L'approche que nous avons adoptée, qui fait suite à d'autres travaux du même type, consistant à dialoguer et à discuter avec une des figures de cette recherche américaine sur le droit, nous semble offrir de riches potentialités pour contribuer à la mise en œuvre d'un renouvellement du régime de connaissance sur le droit.

Nous avons par ailleurs opéré ce travail dans un processus analogue à celui de sociologues, rompant avec l'enfermement dans une sociologie spécialisée, pour retrouver le positionnement des grandes figures fondatrices pour lesquelles le droit était un analyseur privilégié des transformations des sociétés. Ce qui nous a réunis, au-delà de nos différences de disciplines, de spécialisations et grâce aux complémentarités que ces différences suggéraient, c'est la certitude que ce qui se jouait dans cette expérience, c'est aussi le dépassement d'une démarche limitant son horizon à une recherche de sens sur le droit en lui-même, pour luimême. La mise en parallèle avec l'évolution des savoirs sur les sciences et techniques, de même que l'intérêt accordé à une approche du droit par le social et à la mise en relation du droit et de la notion de domination soulignant l'importance de la dimension politique du droit participent d'un renouvellement d'ampleur du regard sur le droit lui-même. Ils nous paraissent aller plus loin encore. Le droit est devenu une entrée à forte potentialité heuristique vers une autre recherche de sens à des niveaux plus généraux : celui qui concerne la construction des savoirs, dont les savoirs en sciences sociales ; celui qui concerne la construction du pouvoir, les formes d'établissement de la domination ou encore les processus de structuration des 
sociétés, leurs régimes de régulation. Par cette entrée, le droit, c'est une véritable exploration des formes de mobilisation des savoirs que nous cherchons à stimuler. 